\title{
Evaluation of Dimensionality Reduction and Truncation Techniques for Word Embeddings
}

\author{
Paulo Henrique Calado Aoun ${ }^{1}$, Andre C. A. Nascimento ${ }^{1}$, Adenilton J. da Silva ${ }^{1}$ \\ ${ }^{1}$ Departamento de Computação \\ Universidade Federal Rural de Pernambuco (UFRPE) \\ Recife - PE - Brasil \\ paulo_calado@live.com, \{andre.camara, adenilton.silva\}@ufrpe.br
}

\begin{abstract}
The use of word embeddings is becoming very common in many Natural Language Processing tasks. Most of the time, these require computacional resources that can not be found in most part of the current mobile devices. In this work, we evaluate a combination of numeric truncation and dimensionality reduction strategies in order to obtain smaller vectorial representations without substancial losses in performance.
\end{abstract}

Resumo. O uso de word embeddings está se tornando muito comum em diversas tarefas de processamento de linguagem natural. Na maioria das vezes, eles exigem recursos computacionais que não podem ser encontrados na maior parte dos dispositivos móveis atuais. Neste trabalho, avaliamos uma combinação de estratégias de truncagem numérica e redução de dimensionalidade para obter representações vetoriais menores sem perdas substanciais no desempenho.

\section{Introdução}

Em diversas tarefas de processamento de linguagem natural (PLN) a identificação do significado de uma palavra é de fundamental importância. Recentemente, a utilização de representações vetoriais, obtidos de forma não supervisionada, tem-se destacado [Levy and Goldberg 2014]. Uma vez que tal vetor seja obtido, é possível calcular a proximidade entre palavras e portanto inferir um significado ou contexto para elas.

No entanto, um fator impactante é o tamanho adotado na representação vetorial, dado que quanto maior o número de dimensões, maior o custo de treinamento. $\mathrm{O}$ mesmo pode se dizer do custo de memória e armazenamento, que também será maior. Tal aspecto é primordial em contextos em que recursos computacionais são ainda mais limitados, como, por exemplo, em dispositivos móveis, e.g. smartphones e tablets. Em [Ling et al. 2016], é demonstrado que 1 milhão de palavras representadas por vetores de 200 dimensões chegam a ocupar 1.6GB de memória em um sistema operacional de 64bits.

Como [Ling et al. 2016] apresenta, a redução da precisão (para binário, 4, 6 e 8 bits) em representações treinadas previamente pode obter resultados similares aos obtidos com precisão de 64 bits em tarefas de similaridade de palavras e de parsers de dependência. Isso mostra que a redução da precisão, não necessariamente representa uma perda substancial no desempenho. Em [Raunak 2017], experimentos foram realizados para avaliar diferentes abordagens de redução de dimensionalidade, obtendo resultados 
equivalentes mesmo quando o número de dimensões de vetores treinados previamente foi reduzido pela metade.

Entretanto, até o persente momento não foram realizados trabalhos que avaliem a combinação destas duas abordagens de compactação de informação. Este trabalho visa avaliar até que ponto a redução de dimensionalidade combinada a truncagem de valores afeta o desempenho de representações vetoriais de palavras em tarefas de similaridade léxica. Mais especificamente, é realizada a combinação de duas técnicas apresentadas em trabalhos anteriores [Raunak 2017, Ling et al. 2016], de diminuição da quantidade de bits (onde por padrão, são utilizados 64) para representação de uma dimensão do vetor [Ling et al. 2016] e de redução de dimensionalidade, utilizando o algoritmo PostProcessing Algorithm (PPA) em conjunto com o algoritmo Principal component analysis (PCA) [Raunak 2017].

Buscamos dessa forma, oferecer uma visão abrangente sobre os limites em que a perda de informação não inviabilize o uso de tais representações. Também apresentamos uma análise da redução do tamanho do arquivo resultante, consequentemente, diminuindo o espaço necessário para utilização do mesmo.

O restante deste trabalho está dividido em 4 seções. A Seção 2 descreve os métodos utilizados neste trabalho. A Seção 3 apresenta os experimentos. Os resultados obtidos são apresentados na Seção 4 e a Seção 5 é a conclusão.

\section{Métodos}

Nesta seção descrevemos as etapas adotadas para a redução de dimensionalidade e de precisão dos vetores de palavras (Figura 2). A primeira etapa consiste na aplicação dos algoritmos de redução de dimensionalidade PCA [Shlens 2014] e PPA [Mu et al. 2017], como proposto em [Raunak 2017]. Este método de redução de dimensionalidade foi escolhido pela sua eficiência em construir word embeddings com menores dimensões, e ainda manter desempenho similar ou até melhor (os autores demonstraram que em 7 dos 12 datasets utilizados no experimento, os word embeddings de 150 dimensões gerados a partir da aplicação do algoritmo, obtiveram resultados em média $2.74 \%$ melhores do que os embeddings de 300 dimensões).

Figura 1. Etapas de pré-processamento de word embeddings.

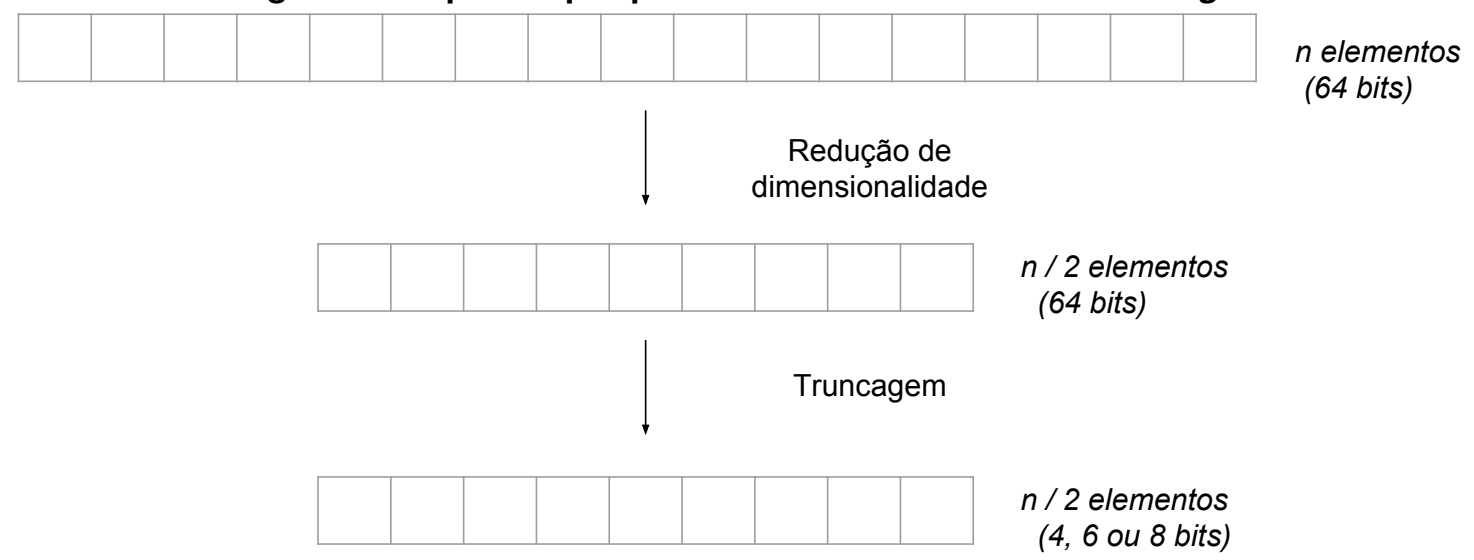

O passo seguinte é a aplicação dos métodos de truncagem propostos por 
[Ling et al. 2016], este método foi escolhido por ser possível reduzir significativamente o número de bits necessário por dimensões em modelos com até 200 dimensões. Os autores demonstraram que 8 bits por dimensão é o suficiente para representação dos valores e manter o desempenho em problemas de similaridade semântica, uma redução significativa em comparação aos 64 bits utilizados em grande parte dos sistemas atuais. Ambos os métodos[Raunak 2017, Ling et al. 2016] são aplicados sobre um conjunto de vetores treinados previamente.

Na seção 2.1, serão detalhados os métodos de redução de dimensionalidade considerados neste trabalho. A seção 2.2 apresenta o algoritmo de truncagem adotado nos experimentos.

\subsection{Redução de dimensionalidade}

O espaço na memória necessário para o armazenamento da representação vetorial das palavras (word embeddings) é diretamente proporcional a dimensionalidade do mesmo. Por este motivo, a redução da dimensionalidade dos vetores se torna essencial para a redução do tamanho do arquivo resultante. As abordagens descritas a seguir são as utilizadas no trabalho para tratar o problema da redução da dimensionalidade.

\section{PCA}

O algoritmo Principal component analysis (PCA) [Shlens 2014] permite reduzir a dimensionalidade de uma base dados através da combinação de atributos em novos atributos (i.e., componentes). Por fim, um subconjunto das componentes geradas pode ser utilizada como uma nova representação (reduzida) dos dados originais. Em [Raunak 2017], é adotado um método de redução de dimensionalidade de embeddings baseado em PCA, o qual descrevemos a seguir:

1. Organizar o dado em uma matriz $n \times m$, onde $n$ é a dimensionalidade dos vetores de palavras e $m$ é o número de palavras do dicionário;

2. Subtrair a média de cada tipo de medida.

3. Aplicar o algoritmo PCA sobre a matriz resultante, e selecionar os $n / 2$ componentes principais.

\section{PPA}

O algoritmo de pós processamento (Post Processing Algorithm, PPA)[Mu et al. 2017] baseia-se em observações geométricas que mostram word embeddings obtidas após a subtração de parte de sua energia (vetor médio), de modo que a informação possa ser representada em um subespaço com menos dimensões. Este algoritmo parte da premissa de que todas as representações de palavras possuem o mesmo vetor comum $u$ e possuem as mesmas direções dominantes $D$. Sendo assim, o vetor comum e as direções influenciam fortemente a representação de palavras da mesma forma, de modo que o algoritmo busca eliminá-los. O algoritmo PPA é apresentado no Algoritmo 1. 
Algoritmo 1: Algoritmo PPA(X,D)

Data: Matriz de Word Embedding X, D

Result: Matriz Word Embedding Pós-processada X

Subtrair a média:

$\mathrm{X}=\mathrm{X}$-mean $(\mathrm{X})$.

Computar componentes PCA:

$u_{i}=\mathrm{PCA}(\mathrm{X})$, onde $\mathrm{i}=1,2, \ldots, \mathrm{d}$

Onde $\mathrm{d}=$ dimensionalidade de embedding

Eliminar maiores componentes: $\forall v$ em $\mathrm{X}$

$v=v-\sum_{i=1}^{D}\left(u_{i}^{T} \times v\right) u_{i}$

Onde $v$ é a representação de uma palavra

\section{Raunak, 2017}

Um outro algoritmo, proposto por [Raunak 2017] consiste em uma pequena modificação no algoritmo PPA. Esta abordagem adota a aplicação do algoritmo PCA em sequência, o que por sua vez leva a uma representação ainda mais compacta em termos de densidade do vetor resultante (Algoritmo 2).

O algoritmo baseia-se em três ideias principais: (i) o algoritmo PPA, comprovadamente resulta em melhores embeddings. (ii) quanto maior o pós-processamento, menor as dimensões dos embeddings, pois projetando de uma forma mais distante os vetores de palavras de suas direções dominantes, é possível gerar melhores embeddings. (iii) A extensão dos principais componentes explica o porque dos dados não serem tão bons como nos embeddings originais de 300 dimensões.

Algoritmo 2: Algoritmo Raunak,2017

Data: Matriz Word Embedding X, Parâmetro Limiar D, Nova Dimensão N

Result: Matriz Word Embedding X com Dimensão reduzida N

Aplicar o Algoritmo de Pós-Processamento:

$\mathrm{X}=\mathrm{PPA}(\mathrm{X}, \mathrm{D})$

Transformar $X$ usando PCA:

$\mathrm{X}=\mathrm{PCA}(\mathrm{X})$

Aplicar o Algoritmo de Pós-Processamento:

$\mathrm{X}=\mathrm{PPA}(\mathrm{X}, \mathrm{D})$

\subsection{Redução do número de bits}

A redução da precisão consiste na truncagem dos valores $x$ em cada posição do vetor de representação de palavras, em uma representação utilizando $n$ bits [Ling et al. 2016]. Por exemplo, para arredondar um valor $x$ que fique dentro de um conjunto de valores $[-r, r]$ onde $r$ é dado por:

$$
r=2^{n-1}-1 .
$$


E a função de arredondamento pode então ser expressa como:

$$
R(x, n)=\left\{\begin{array}{l}
\lfloor x\rfloor, \quad \text { se } \quad x \leq\lfloor x\rfloor+\frac{\epsilon}{2} \\
\lfloor x\rfloor+\epsilon,
\end{array}\right.
$$

com $\epsilon$ sendo definido como:

$$
\epsilon=2^{1-n} r .
$$

\section{Experimentos}

Os experimentos foram realizados no contexto de similaridade semântica de palavras, utilizando os benchmarks resumidos por [Faruqui and Dyer 2014]. Cada base de dados é composta por pares de palavras, cuja similaridade foi avaliada por humanos. Ao avaliar vetores de palavras, a similaridade entre as palavras é calculada pelo cosseno de suas representações vetoriais. Em seguida, calcula-se o coeficiente de correlação de Spearman [Well and Myers 2003] entre as classificações produzidas automaticamente e as anotadas manualmente. Desse modo, quanto maior a similaridade entre palavras, maior o valor da métrica de avaliação.

Os experimentos foram realizados utilizando os vetores GloVe [Pennington et al. 2014] pretreinados, de 300 dimensões treinados sobre o dump do Wikipedia (2014) + Gigaword 5 (6B tokens, 400K palavras). Em todos os experimentos realizados, aplicou-se o critério de redução para metade da dimensionalidade original, i.e., foram produzidos vetores com 150 dimensões.

Para avaliar e comparar os efeitos das diferentes estratégias de redução de dimensionalidade [Raunak 2017] e a truncagem, foram considerados os seguintes algoritmos:

1. PCA: Transforma os vetores de palavras utilizando o algoritmo PCA.

2. P-PCA: Transforma os vetores de palavras utilizando o algoritmo PCA após a aplicação do algoritmo de pós processamento.

3. PCA-P: Transforma os vetores de palavras utilizando o algoritmo PCA e então aplica o algoritmo de pós processamento.

4. RAUNAK: Transforma os vetores de palavras utilizando o algoritmo proposto por [Raunak 2017]

Os embeddings resultantes foram então truncados considerando 4, 6 e 8 bits, conforme descrito na Seção 2.2. Nos experimentos realizados, foram utilizadas as implementações do PCA disponível na ferramenta Scikit-Learn [Pedregosa et al. 2011]. As implementações dos algoritmos PPA e RAUNAK consideraram as versões disponibilizadas pelos próprios autores [Raunak 2017] .

\section{Resultados}

Os resultados individuais para cada combinação de método de redução de dimensionalidade e truncagem são apresentados na Tabela 1. Com exceção do Glove-300 mostrado na tabela, todos os outros arquivos foram reduzidos de 300, para 150 dimensões. Podemos observar que em algumas bases de dados, como por exemplo, o EN-MC-30, a aplicação do algoritmo PPA seguido do PCA, com truncagem de 4 bits apresentou desempenho melhor do que a representação original. O mesmo pode ser observado na base 
EN-VERB-143, na qual o algoritmo RAUNAK com truncagem de 6 bits também obteve o melhor desempenho.

Através da tabela é possível perceber que dentre as três truncagens utilizadas ( 4,6 e 8 bits), a truncagem de 4 bits obteve em geral melhores resultados, apresentando desempenho mais próximo (ou superior), ao original em 33 dos 52 experimentos. A truncagem de 8 bits, apresentada por [Ling et al. 2016] como suficiente para manter a performance em seu trabalho, quando combinada à estratégias de redução de dimensionalidade obteve a performance mais próxima da original em apenas 5 dos experimentos, sendo 4 desses obtidos através do algoritmo PCA. Nos outros 14 experimentos restantes, a truncagem de 6 bits foi a que mais se aproximou (ou obteve melhor desempenho). Esses resultados indicam que a diminuição no número de dimensões do vetor de representação vetorial, impacta diretamente na redução do número de bits necessários para uma efetiva codificação de informação semântica.

Por outro lado, a redução do tamanho dos arquivos contendo as representações finais chega a ser bastante significativa, conforme apresentado na Figura 4, na qual se demonstra a correlação entre o tamanho do arquivo final e o desempenho médio (sobre todas as bases de dados analisadas) para cada combinação de método + truncagem.

Como esperado, os melhores resultados na redução de tamanho foram obtidos nos arquivos truncados com 4 bits. No melhor caso, onde o PCA foi utilizado após a aplicação do PPA, obtivemos uma redução de aproximadamente $40.73 \%$ no tamanho do arquivo, e uma perda média de apenas $6.39 \%$ na correlação de Spearman com as avaliações feitas por humanos. De maneira geral, o algoritmo proposto por [Raunak 2017] e a abordagem PPA-PCA obtiveram as maiores reduções de tamanho do arquivo $(40,80 \%$ menores que o original). Apesar disso, é possível observar na Tabela 2 que as porcentagens de redução do tamanho do arquivo se mantiveram bastante semelhantes em todas as abordagens. A Tabela 2 também possui a diferença do desempenho médio (Correlação Spearman) dos métodos em cada base. A truncagem de 4 bits, em sua maioria, também resultou numa menor diferença em relação a média da Correlação Spearman dos arquivos sem truncagem.

\section{Conclusão}

Os experimentos realizados complementam os estudos anteriores, ao combinar análises de redução de dimensionalidade com técnicas de redução de precisão dos vetores de word embeddings pré-treinados. Observou-se que a utilização da redução de dimensionalidade em conjunto com a redução da quantidade de bits necessária para representação de um vetor, de fato reduz significantemente o tamanho do arquivo resultante sem sacrificar muito a performance.

Esperamos que os resultados apresentados neste trabalho sirvam como referência para escolha de representações vetoriais de palavras para uso em dispositivos de memória reduzida e limitado poder computacional. Como trabalhos futuros, esperamos avaliar outras estratégias de redução de dimensionalidade, bem como avaliar os efeitos da redução de precisão durante o treinamento dos vetores, conforme indicado em [Ling et al. 2016].

Outro possível trabalho futuro é representar os vetores de word embeddings utilizando um sistema quântico [Nielsen and Chuang 2002]. Teoricamente a capacidade de 
Tabela 1. Resultados individuais de cada arquivo de configuração

\begin{tabular}{|c|c|c|c|c|c|}
\hline Dataset & Embedding & Sem truncagem & 4 bits & 6 bits & 8 bits \\
\hline \multirow[t]{5}{*}{ EN-MC-30 } & Glove & 0.7026 & 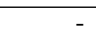 & - & \\
\hline & PCA & 0.6934 & 0.6554 & 0.6569 & 0.5690 \\
\hline & PCA-PPA & 0.6917 & 0.6156 & 0.6289 & 0.5882 \\
\hline & PPA-PCA & 0.7144 & 0.7255 & 0.6841 & 0.6899 \\
\hline & RAUNAK & 0.7195 & 0.7177 & 0.5906 & 0.5788 \\
\hline \multirow[t]{5}{*}{ EN-MEN-TR-3k } & Glove & 0.7375 & - & - & \\
\hline & PCA & 0.6251 & 0.5516 & 0.5505 & 0.5429 \\
\hline & PCA-PPA & 0.7491 & 0.6911 & 0.6969 & 0.6767 \\
\hline & PPA-PCA & 0.7539 & 0.7079 & 0.6847 & 0.6917 \\
\hline & RAUNAK & 0.7531 & 0.7163 & 0.6943 & 0.6916 \\
\hline \multirow[t]{5}{*}{ EN-MTurk-287 } & Glove & 0.6332 & & & \\
\hline & PCA & 0.5429 & 0.5000 & 0.5443 & 0.4734 \\
\hline & PCA-PPA & 0.6266 & 0.6053 & 0.6219 & 0.5949 \\
\hline & PPA-PCA & 0.6594 & 0.6261 & 0.5384 & 0.6023 \\
\hline & RAUNAK & 0.6199 & 0.5904 & 0.5700 & 0.5935 \\
\hline \multirow[t]{5}{*}{ EN-MTurk-771 } & Glove & 0.6501 & & & \\
\hline & PCA & 0.5263 & 0.4701 & 0.4571 & 0.4596 \\
\hline & PCA-PPA & 0.6461 & 0.5843 & 0.5452 & 0.5745 \\
\hline & PPA-PCA & 0.6491 & 0.5791 & 0.5905 & 0.5442 \\
\hline & RAUNAK & 0.6483 & 0.6312 & 0.5572 & 0.5567 \\
\hline \multirow[t]{5}{*}{ EN-RG-65 } & Glove & 0.7662 & & & \\
\hline & PCA & 0.7283 & 0.6447 & 0.6896 & 0.6028 \\
\hline & PCA-PPA & 0.7087 & 0.6956 & 0.6683 & 0.5066 \\
\hline & PPA-PCA & 0.7501 & 0.7007 & 0.6647 & 0.6717 \\
\hline & RAUNAK & 0.7784 & 0.6790 & 0.6034 & 0.6045 \\
\hline \multirow[t]{5}{*}{ EN-RW-STANFORD } & Glove & 0.4118 & & & \\
\hline & PCA & 0.2839 & 0.2399 & 0.232 & 0.2244 \\
\hline & PCA-PPA & 0.4166 & 0.3605 & 0.3428 & 0.3387 \\
\hline & PPA-PCA & 0.4397 & 0.3701 & 0.3247 & 0.3375 \\
\hline & RAUNAK & 0.4354 & 0.3712 & 0.3635 & 0.3529 \\
\hline \multirow[t]{5}{*}{ EN-SIMLEX-999 } & Glove & 0.3705 & & & \\
\hline & PCA & 0.2838 & 0.2142 & 0.1939 & 0.1959 \\
\hline & PCA-PPA & 0.3591 & 0.2979 & 0.2932 & 0.2786 \\
\hline & PPA-PCA & 0.3616 & 0.3215 & 0.3041 & 0.3173 \\
\hline & RAUNAK & 0.3767 & 0.3405 & 0.3441 & 0.3158 \\
\hline \multirow[t]{5}{*}{ EN-SimVerb-3500 } & Glove & 0.2267 & & & \\
\hline & PCA & 0.1317 & 0.0974 & 0.0793 & 0.0776 \\
\hline & PCA-PPA & 0.2525 & 0.1806 & 0.2065 & 0.1898 \\
\hline & PPA-PCA & 0.2448 & 0.2065 & 0.2019 & 0.1772 \\
\hline & RAUNAK & 0.2643 & 0.2310 & 0.2228 & 0.1829 \\
\hline \multirow[t]{5}{*}{ EN-VERB-143 } & Glove & 0.3051 & - & - & - \\
\hline & PCA & 0.2883 & 0.1923 & 0.1636 & 0.2278 \\
\hline & PCA-PPA & 0.3848 & 0.2013 & 0.3722 & 0.3043 \\
\hline & PPA-PCA & 0.367 & 0.2748 & 0.3940 & 0.2161 \\
\hline & RAUNAK & 0.4012 & 0.3496 & 0.4634 & 0.3213 \\
\hline \multirow[t]{5}{*}{ EN-WS-353-ALL } & Glove & 0.6054 & - & - & - \\
\hline & PCA & 0.4777 & 0.3531 & 0.3466 & 0.3917 \\
\hline & PCA-PPA & 0.6652 & 0.6055 & 0.5722 & 0.5771 \\
\hline & PPA-PCA & 0.6648 & 0.6513 & 0.5926 & 0.6136 \\
\hline & RAUNAK & 0.6632 & 0.6125 & 0.5865 & 0.5911 \\
\hline \multirow[t]{5}{*}{ EN-WS-353-REL } & Glove & 0.5725 & - & - & - \\
\hline & PCA & 0.4272 & 0.3223 & 0.2982 & 0.3541 \\
\hline & PCA-PPA & 0.6096 & 0.545 & 0.5093 & 0.5141 \\
\hline & PPA-PCA & 0.6085 & 0.6005 & 0.5329 & 0.555 \\
\hline & RAUNAK & 0.6085 & 0.5491 & 0.5101 & 0.5153 \\
\hline \multirow[t]{5}{*}{ EN-WS-353-SIM } & Glove & 0.6638 & & - & - \\
\hline & PCA & 0.5469 & 0.4149 & 0.4253 & 0.4562 \\
\hline & PCA-PPA & 0.7128 & 0.6545 & 0.6455 & 0.6450 \\
\hline & PPA-PCA & 0.7250 & 0.7010 & 0.6432 & 0.6744 \\
\hline & RAUNAK & 0.7189 & 0.6615 & 0.6397 & 0.6429 \\
\hline \multirow[t]{5}{*}{ EN-YP-130 } & Glove & 0.5613 & & & \\
\hline & PCA & 0.4333 & 0.3188 & 0.2607 & 0.2850 \\
\hline & PCA-PPA & 0.5516 & 0.4004 & 0.4260 & 0.4245 \\
\hline & PPA-PCA & 0.5693 & 0.5632 & 0.3997 & 0.5080 \\
\hline & RAUNAK & 0.5968 & 0.4322 & 0.3899 & 0.3673 \\
\hline
\end{tabular}


Tabela 2. Tamanho do arquivo e diferença do desempenho médio (Correlação Spearman) dos métodos em cada base

\begin{tabular}{l|l|r|r|r} 
Embedding & Truncagem & Tamanho arquivo (MB) & Redução do arquivo $\%$ & Diferença Spearman \\
\hline PCA & Sem truncagem & 573,9 & - & - \\
& 4 bits & 340,1 & 40,74 & $>16,93 \%$ \\
& 6 bits & 396,8 & 30,86 & $>18,21 \%$ \\
& 8 bits & 454,8 & 20,75 & $>18,84 \%$ \\
\hline P-PCA & Sem truncagem & 573,8 & - & - \\
& 4 bits & 340,1 & 40,73 & $>6,39 \%$ \\
& 6 bits & 396,8 & 30,85 & $>12,68 \%$ \\
& 8 bits & 454,8 & 20,74 & $>12,10 \%$ \\
\hline PCA-P & Sem truncagem & 573,8 & - & - \\
& 4 bits & 339,7 & 40,80 & $>12,70 \%$ \\
& 6 bits & 396,7 & 30,86 & $>11,46 \%$ \\
& 8 bits & 454,6 & 20,77 & $>15,75 \%$ \\
\hline RAUNAK & Sem truncagem & 573,8 & - & - \\
& 4 bits & 339,7 & 40,80 & $>9,26 \%$ \\
& 6 bits & 396,6 & 30,88 & $>13,82 \%$ \\
& 8 bits & 454,8 & 20,74 & $>16,74 \%$ \\
\hline
\end{tabular}

Figura 2. Gráfico relacionando desempenho médio (linha vermelha) e o tamanho do arquivo com embeddings.

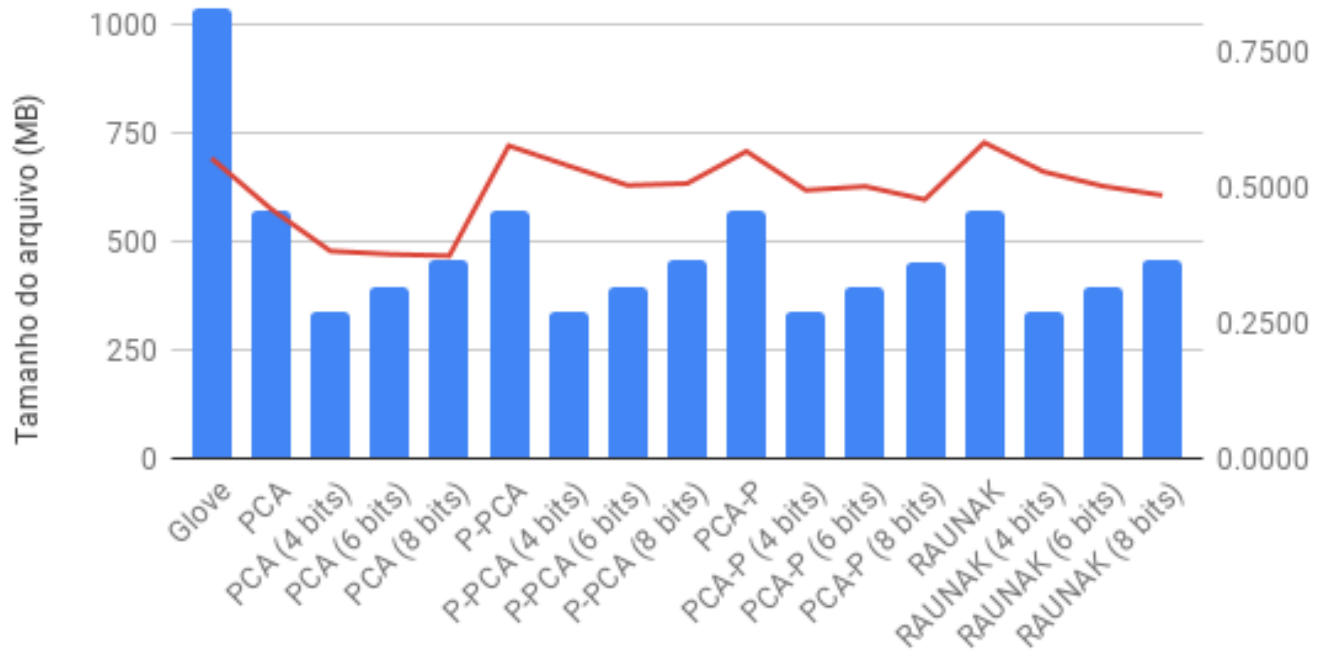

Método

armazenamento de informação em sistemas quânticos possui um ganho exponencial em relação aos computadores convencionais [Trugenberger 2001].

\section{Agradecimentos}

Este trabalho recebeu apoio do Instituto Serrapilheira (número do processo Serra-170922626).

\section{Referências}

Faruqui, M. and Dyer, C. (2014). Community evaluation and exchange of word vectors. Proceedings of 52nd Annual Meeting of the Association for Computational Linguistics: 
System Demonstrations., pages 19-24.

Levy, O. and Goldberg, Y. (2014). Dependency-based word embeddings. Proceedings of the 52nd Annual Meeting of the Association for Computational Linguistics (Volume 2: Short Papers), pages 302-308.

Ling, S., Song, Y., and Roth, D. (2016). Word Embeddings with Limited Memory. In Proceedings of the 54th Annual Meeting of the Association for Computational Linguistics, pages 387-392.

$\mathrm{Mu}$, J., Bhat, S., and Viswanath, P. (2017). All-but-the-top: Simple and effective postprocessing for word representations. arXiv preprint arXiv:1702.01417.

Nielsen, M. A. and Chuang, I. (2002). Quantum computation and quantum information.

Pedregosa, F., Varoquaux, G., Gramfort, A., Michel, V., Thirion, B., Grisel, O., Blondel, M., Prettenhofer, P., Weiss, R., Dubourg, V., and Others (2011). Scikit-learn: Machine learning in Python. Journal of machine learning research, 12(Oct):2825-2830.

Pennington, J., Socher, R., and Manning, C. (2014). Glove: Global vectors for word representation. In Proceedings of the 2014 conference on empirical methods in natural language processing (EMNLP), pages 1532-1543.

Raunak, V. (2017). Effective dimensionality reduction for word embeddings. arXiv preprint arXiv:1708.03629.

Shlens, J. (2014). A tutorial on principal component analysis. arXiv preprint arXiv:1404.1100.

Trugenberger, C. A. (2001). Probabilistic quantum memories. Physical Review Letters, 87(6):067901.

Well, A. D. and Myers, J. L. (2003). Research design \& statistical analysis. Psychology Press. 